\title{
Molecular epidemiology of canine parvovirus in southern India
}

\author{
V. M. Vivek Srinivas ${ }^{1}$, H. K. Mukhopadhyay ${ }^{1}$, J. Thanislass' ${ }^{2}$, P. X. Antony ${ }^{1}$ and R. M. Pillai ${ }^{1}$ \\ 1. Department of Microbiology, Rajiv Gandhi College of Veterinary and Animal Sciences, \\ Puducherry - 605 009, India; 2. Department of Veterinary Biochemistry, Rajiv Gandhi College of Veterinary \\ and Animal Sciences, Puducherry - 605 009, Iindia \\ Corresponding author: V. M. Vivek Srinivas, Present address: FMD Vaccine Production Lab, Indian Veterinary Research \\ Institute (IVRI), Hebbal, Bangalore-560 024. Ph: +9197428 91992, email: vivekvet24@gmail.com \\ Received: 29-05-2013, Revised: 02-07-2013, Accepted: 03-07-2013, Published online: 13-08-2013
}

doi: 10.14202/vetworld.2013.744-749

How to cite this article: Srinivas VMV, Mukhopadhyay HK, Thanislass J, Antony PX and Pillai RM (2013) Molecular epidemiology of canine parvovirus in southern India, Veterinary World 6(10): 744-749.

\begin{abstract}
Aim: The present study was conducted to isolate and characterize canine parvovirus circulating in Southern India by genetic analysis of VP2 capsid protein gene.

Materials and Methods: In this study, 128 samples were collected from nine different locations covering five Southern Indian states (Pondicherry, Tamil Nadu, Kerala, Andhra Pradesh and Karnataka). Out of 128 samples, 69 samples were found to be positive by PCR assay. Out of 69 positive samples, 36 were randomly selected and processed for virus isolation. Twenty viruses could be isolated successfully and 18 randomly selected isolate were subjected to VP2 gene sequence analysis along with 6 random clinical samples.

Result: Seventeen isolates and 5 clinical samples were characterized as New CPV-2a (CPV2a with 297-Ser $\rightarrow$ Ala). But one isolate and one clinical sample had amino acids variations which were characteristics of New CPV-2b. The phylogenetic analysis revealed that one of the field isolates was found to be phylogenetically closely related to New CPV-2b strains of India; rest other sequences was found to share ancestral origins with New CPV-2a reference strains of Japan, China, Thailand and India.
\end{abstract}

Conclusion: The present study revealed that the predominant CPV strain circulating in Southern India is New CPV-2a. There is also enough indication of New CPV-2b strain from different states of Southern India.

Keywords: Canine parvovirus (CPV), crandell feline kidney (CRFK) cell line, molecular epidemiology, phylogenetic analysis, sequence analysis.

\section{I ntroduction}

Canine parvovirus (CPV) is the most significant viral cause of acute haemorrhagic enteritis and myocarditis in puppies over the age of 3-4 months [1]. CPV presumably originated as a host range variant from feline panleukopenia virus (FLV) that adapted to new canine host via wild carnivores like minks and foxes [2]. A small, round non-enveloped virus was observed by electron microscopy in stool specimens and tissues in affected animals. Subsequently, a novel parvovirus was isolated in both canine and feline cell cultures [3]. The Canine parvovirus 2 (CPV-2) belongs to the family Parvoviridae with the genome of linear single stranded DNA of $5.2 \mathrm{~kb}$ in size. The CPV genome has two open reading frames (ORF) of which the first ORF encodes one non structural protein (NS1) and the second ORF encodes two capsid proteins (VP1 and VP2) which are translated from alternatively spliced mRNA [4]. VP2 gene mainly comprises the icosahedral capsid of CPV, and only a few amino acid substitutions in its sequence can alter relevant immunological characteristics of the virus $[5,6,7]$.

Canine parvovirus (CPV) caused by CPV-2

Copyright: The authors. This article is an open access article licensed under the terms of the Creative Commons Attribution License (http://creativecommons.org/licenses/by/2.0) which permits unrestricted use, distribution and reproduction in any medium, provided the work is properly cited. emerged in 1978 as a highly contagious and very serious disease in dogs [1]. But CPV-2 underwent evolutionary changes after its emergence in the late 1970s and within few years CPV-2 has been globally replaced by the newer antigenic variants viz., CPV-2a and CPV-2b [1, 4, 8]. At present, the variants of CPV are distributed worldwide and the original prototype 2 no longer circulates in the dog population [9, 10, 11]. In 2000, a new variant of CPV which was named as CPV-2c was reported in Italy [12]. This new variant was distinguishable from CPV $2 a / 2 b$ by the substitution of Glu in lieu of Asn/Asp at 426 residue of the VP2 capsid protein; therefore it was also referred to as Glu-426 mutant [12]. Additional amino acid difference was observed in both CPV-2a and CPV-2b at position 297 (Ser to Ala) [10]. This mutation was first appeared in 1993 in German CPV isolates and was designated as New CPV-2a/2b [10].

Molecular diagnostic techniques like PCR based methods had been the most reliable techniques having high degree of sensitivity and specificity in detecting CPV from faecal samples [12]. Amplification of VP2 gene fragment (capsid protein) using primers like $\mathrm{H}_{\mathrm{for}} / \mathrm{H}_{\mathrm{rev}}$ and subsequent sequencing of the PCR products covering the informative amino acids would definitely help in detecting genetic variation which exists between CPV-2 and its variants [12]. 
Most commercial vaccines available in India contain CPV-2 strain whereas; only one CPV-2b based vaccine is available [13]. Therefore, further epidemiological survey and molecular characterization of CPV involving samples from larger geographical area would be extremely helpful in formulating a suitable vaccination strategy.

Hence, a study was planned on molecular survey and characterization of canine parvovirus in Southern India using PCR and subsequent sequencing of the PCR products covering a portion of VP2 gene (capsid protein). The genetic analysis of canine parvovirus strains in dog population of various states in Southern India will reveal the predominant CPV strain/strains responsible for causing outbreaks in a larger geographical area.

\section{Materials and Methods}

Clinical samples: A total of 128 faecal samples/rectal swabs were collected from the dogs suspected to be suffering from diarrhoea/enteritis due to canine parvovirus infection from nine different cities namely Puducherry (Pondicherry), Chennai, Coimbatore (Tamil Nadu), Hyderabad, Tirupati (Andhra Pradesh), Bangalore (Karnataka), Thrissur, Palakad and Trivandrum (Kerala) covering all the Southern Indian states, India, during a period of 11 months from April 2011 to February 2012 (Table-1). The collected samples were emulsified in $1 \mathrm{ml}$ of $0.1 \mathrm{M}$ PBS of $\mathrm{pH}$ 7.4 and centrifuged at $6000 \mathrm{rpm}$ for $15 \mathrm{~min}$ at $4^{\circ} \mathrm{C}$. The supernatant was collected and used for PCR amplification and for virus isolation [13].

Template DNA preparation: Hundred microlitres of the processed supernatant was used for template DNA preparation by boiling at $96^{\circ} \mathrm{C}$ for $10 \mathrm{~min}$ and chilling immediately in crushed ice [14]. The supernatants were diluted 1:10 in distilled water to reduce residual inhibitors of DNA polymerase activity [15].

Primer pair and PCR amplification: The processed samples were screened by primer pair $\mathrm{H}$-for $\left(5^{\prime}\right.$ CAGGTGATGAAT TTGCTACA-3') and H-rev (5'CATTTGGATAAACTGGTGGT-3') that amplifies $630 \mathrm{bp}$ fragment of the gene encoding capsid protein. PCR amplification was carried out as per Buonavoglia [12] and the products were analyzed by electrophoresis using $1.5 \%$ agarose gel in Tris acetate EDTA (TAE) buffer (1X).

Buonavoglia designed the primer pair $\mathrm{H}_{\mathrm{for}} / \mathrm{H}_{\mathrm{rev}}$ and utilized them for sequencing studies of canine parvovirus since this primer pair amplifies a portion of VP2 gene containing the critical amino acids such as 297, 300, 305, 375 and 426, which helps in characterization of canine parvovirus [12].

Virus isolation: Thirty six processed clinical samples, representing all the nine different locations which were positive by PCR are randomly selected were filtered using $0.22 \mathrm{~mm}$ membrane filter (Millipore) and the filtrates were used for virus isolation in 70-75\% CRFK cell line monolayer. Virus isolation was carried out as per the procedure recommended by Hirayama [16]. The infected monolayers were harvested at the 4th day of post infection (with or without CPE) by three cycles of alternative freezing and thawing. The virus supernatants were screened for the presence of virus by PCR using the same primer pair $\mathrm{H}_{\text {for }}$ and $\mathrm{H}_{\text {rev }}$.

Sequencing and phylogenetic analysis: The amplified PCR products of 18 randomly selected cell culture isolates and six randomly selected clinical samples were gel extracted and custom sequenced with primer pair Hfor/Hrev using the automated sequencer, Applied Biosystem 3100. The specificity of the sequences obtained, the nucleotide variations and amino acid variations with respect to the VP2 gene sequence of canine parvovirus were determined using BLAST [Basic Local Alignment Search Tool] (http://blast.ncbi.nlm.nih.gov/Blast.cgi) [13]. The nucleotide sequences of VP2 gene were aligned with sequences of prototype CPV strains (M38246- FPV; M38245-CPV-2; M24003-CPV-2a; M74849-CPV-2b; AY742953-New CPV-2a; AY742955-New CPV-2b; FJ222821- CPV-2c) using ClustalW (www.ebi.ac.uk/ clustalw) and analyzed for the nucleotide variation of VP2 gene at positions 3675, 3685, 3699, 3909 and 4064 with the corresponding amino acid residues at 297, 300, 305, 375 and 426, respectively [13].

For phylogenetic analysis, 30 canine parvovirus sequences from various parts of the world were retrieved from the GenBank and used. The sequences were aligned using ClustalW 1.8 program and .aln and .nxs files were generated. The aln file was converted to .meg file using Mega4.1 [17] and Neighbor Joining tree $(\mathrm{NJ}$ tree) was constructed (bootstrap replicates $=$ 1000; seed $=64,248$ ) using Kimura 2 parameter method for pairwise deletion at uniform rates [13].

The CPV sequences of 18 cell culture isolates and 6 clinical samples under study were submitted in GenBank under the accession numbers JF900758, JF900759，JF900760，JF900761，JF900762, JN008380，JN008381，JN008382，JN008383， JN008384，JN008385，JN008386，JN008387, JN008388，JN008389，JN008390，JN008391, JN008392，JN008393， JN008394， JN008395, JN008396, JN008397 and JN008398 (Table-1).

\section{Results and Discussion}

Out of 128 samples screened, 69 (53.90\%) samples were found to be positive by PCR assay using $\mathrm{H}$ primer. The disease was predominantly noticed in dogs between 0-6 months (73.91\%) as also reported by other authors [13, 18, 19]. It was well known that increased intestinal epithelial turnover caused by changes in the microflora, diet (weaning) and diminishing maternal antibody level were the predisposing factors to CPV infection in pups [20]. The occurrence of CPV enteritis, in this study, was found to be more in males $(62.31 \%)$ in comparison to females $(37.68 \%)$. High percentage of occurrence of CPV 
Table-1. Details of occurrence of CPV based on location (southern India)

\begin{tabular}{llccl}
\hline State & Place & $\begin{array}{c}\text { No. of clinical } \\
\text { samples collected }\end{array}$ & $\begin{array}{c}\text { No. of samples positive } \\
\text { by PCR assay } \mathbf{( H}_{\text {for }} / \mathbf{H}_{\text {rev }} \text { ) }\end{array}$ & $\begin{array}{l}\text { Sequences submitted in Genbank } \\
\text { (Accession numbers) }\end{array}$ \\
\hline Pondicherry & Pondicherry & 25 & 11 & JN008387, JN008388, JN008389 \\
Tamil Nadu & Chennai & 20 & 12 & JF900758, JN008383, JN008397 \\
& Coimbatore & 10 & 8 & JN008384, JN008396 \\
Kerala & Thrissur & 10 & 4 & JN008386 \\
& Palakkad & 10 & 6 & JF900762*, JN008385 \\
& Trivandrum & 10 & 3 & JN008392 \\
Andhra Pradesh & Hyderabad & 10 & 7 & JF900761, JN008393*, JN008398 \\
Karnataka & Tirupati & 15 & 9 & JF900759, JN008390, JN008391 \\
\multirow{2}{*}{ Total } & Bangalore & 18 & 9 & JF900760, JN008380, JN008381, \\
& & & $69(53.90 \%)$ & JN008382, JN008394, JN008395 \\
\hline
\end{tabular}

* Nucleotide variations were characteristics of New CPV-2b (CPV-2b with amino acid variation 297Ser $\rightarrow$ Ala). Other Sequences were characteristics of New CPV-2a (CPV-2a with amino acid variation $297 \mathrm{Ser} \rightarrow$ Ala)

Table-2. Details of occurrence of CPV based on age, sex and vaccination status

\begin{tabular}{lcc}
\hline Status & No. of samples collected & No. of samples positive by PCR assay \\
\hline $0-6$ months & 88 & $51(73.91 \%)$ \\
$6-12$ months & 29 & $13(18.84 \%)$ \\
Above 12 months & 11 & $5(7.24 \%)$ \\
Male & 77 & $43(62.31 \%)$ \\
Female & 51 & $26(37.68 \%)$ \\
Non-vaccinated & 96 & $58(84.05 \%)$ \\
Vaccinated & 32 & $11(15.94 \%)$ \\
\hline
\end{tabular}

Table-3. Nucleotides and amino acid variation in the VP2 protein of different CPV-2

\begin{tabular}{llllll}
\hline CPV Strains & \multicolumn{5}{c}{ Nucleotides in CPV Genome } \\
\cline { 2 - 6 } & $\mathbf{3 6 7 6}$ & $\mathbf{3 6 8 5}$ & $\mathbf{3 6 9 9}$ & $\mathbf{3 9 0 9}$ & $\mathbf{4 0 6 2}$ \\
\hline Amino acid residue in VP2 & 297 & 300 & 305 & 375 & 426 \\
CPV-2 & TCT/Ser & GCT/Ala & GAT/Asp & AAT/Asn & AAT/Asn \\
CPV-2a & TCT/Ser & GGT/Gly & TAT/Tyr & GAT/Asp & AAT/Asn \\
CPV-2b & TCT/Ser & GGT/Gly & TAT/Tyr & GAT/Asp & GAT/Asp \\
New CPV-2a & GCT/Ala & GGT/Gly & TAT/Tyr & GAT/Asp & AAT/Asn \\
New CPV-2b & GCT/Ala & GGT/Gly & TAT/Tyr & GAT/Asp & GAT/Asp \\
CPV-2c & GCT/Ala & GGT/Gly & TAT/Tyr & GAT/Asp & GAAGlu \\
\hline
\end{tabular}

infection in non-vaccinated animals $(84.05 \%)$ indicated that current vaccines confer reasonably good protection $[2,21]$, though there are few reports of vaccinated animals coming down with CPV infection indicating vaccine failure $[13,19,20]$ (Table-2).

Isolation of virus was attempted in CRFK cell line from 36 samples found positive by PCR assay representing the diverse locations of collection. Twenty samples showed mild cytopathic effects in the form of rounding, increased granularity and detached cells, from the third passage level onwards. Moderate isolation rate $(55.55 \%)$ in this study may be attributable to the presence of antibodies in the intestinal lumen of the infected dogs, which may bind virions and prevent viral attachment to cell receptors. Similar observations were also made by Decaro and Mohanraj who observed that the isolation of CPV could be done only for few days of post infection $[13,14]$. Cryolysates of all the twenty samples were confirmed positive by PCR assay using $\mathrm{H}_{\mathrm{for}} / \mathrm{H}_{\mathrm{rev}}$ primer pair at the third passage level.

In this study, the amplified PCR products of eighteen cell culture isolates and six clinical samples were gel extracted and custom sequenced using Automated Sequencer, Applied Biosciences 3100. The sequences obtained were subjected to 'BLAST' to study specificity and strain identity. Sequences obtained were found to be highly specific to CPV as indicated by the maximum identity (95-99\%) obtained with VP2 gene sequence of other canine parvovirus strains available in the GenBank.

The partial VP2 gene of 24 sequences was aligned with the reference strains for sequence analysis. In comparison to prototype CPV-2 (CPV b, M38245), the cell culture isolates and the clinical samples under study had nucleotide variations at position $3675(\mathrm{~T} \rightarrow$ $\mathrm{G}) ; 3685(\mathrm{C} \rightarrow \mathrm{G}) ; 3698(\mathrm{G} \rightarrow \mathrm{T}) ; 3908(\mathrm{~A} \rightarrow \mathrm{G})$ and identical nucleotide (A) at position 4062 (Table 2). Seventeen cell culture isolates and five clinical samples had amino acids Ala, Gly, Tyr, Asp and Asn at residues $297,300,305,375$ and 426 respectively. These nucleotide variations were characteristics of New CPV-2a (CPV-2a with nucleotide variation $\mathrm{T} \rightarrow \mathrm{G}$ at position 3675 or CPV-2a with amino acid variation 297-Ser $\rightarrow$ Ala). In one cell culture isolate JN008393 (Hyderabad) and one clinical sample JF900762 (Palakkad) had amino acids Ala, Gly, Tyr, Asp and Asp at residues $297,300,305,375$ and 426 respectively. These nucleotide variations were characteristics of New CPV-2b (CPV-2b with nucleotide variation $\mathrm{T} \rightarrow \mathrm{G}$ at position 3675 or CPV-2b with amino acid variation 297-Ser $\rightarrow$ Ala).

Twenty two out of 24 sequences had a nucleotide variations which were characteristics of New CPV-2a, was also reported by other authors $[10,13,15,22]$. This 


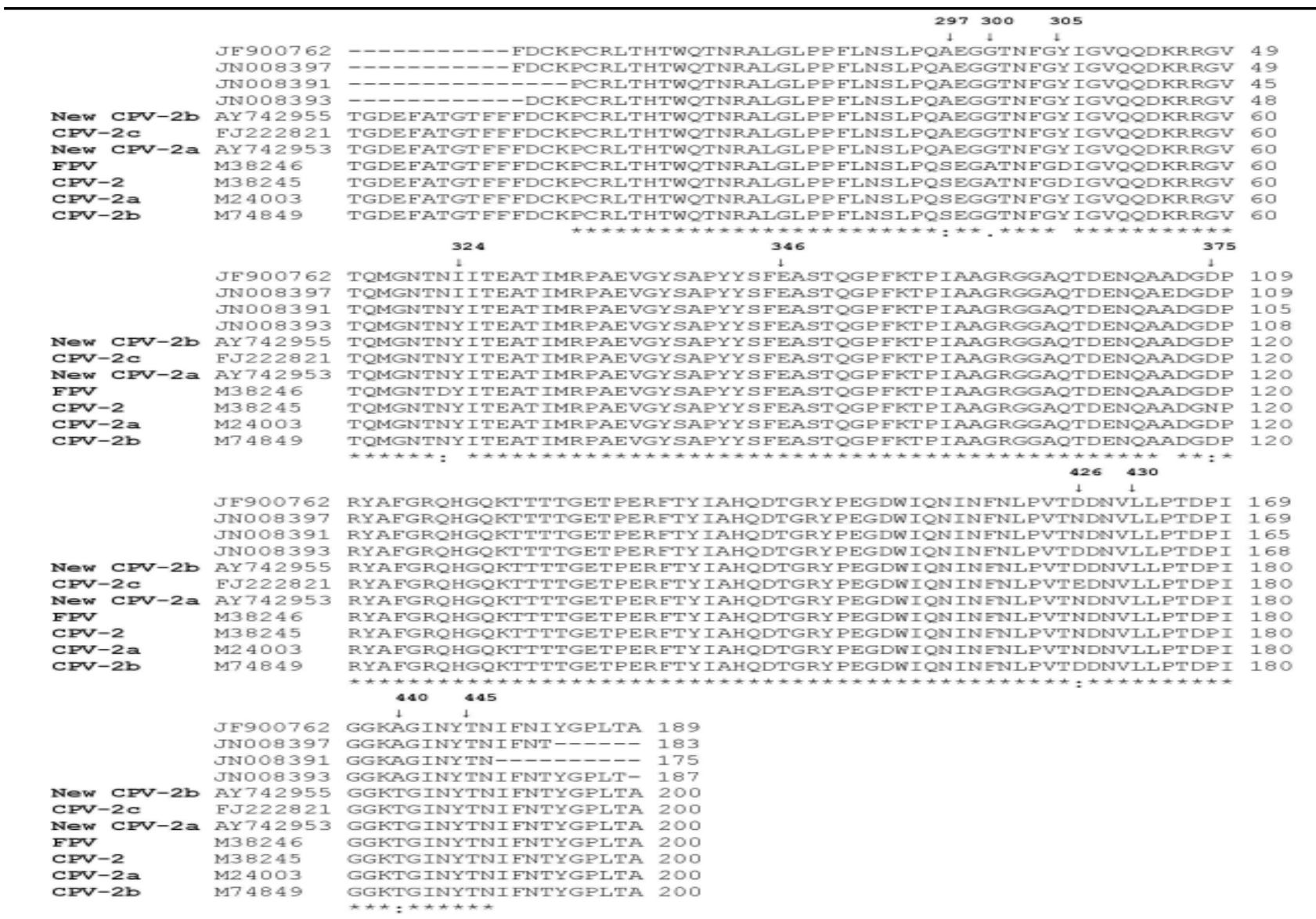

Figure-1. Alignment of the deduced amino acid sequences of partial VP2 gene. Sequence of the representative canine parvovirus field isolate/sample J N008391, J N008395 (New CPV 2a), J N008393, J F900762 (New CPV-2b) is shown aligned with the reference strains M38246 (FPV), M38245 (CPV-2), M24003 (CPV-2a), M74849 (CPV-2b), AY742953 (New CPV-2a), AY742955 (New CPV-2b) and FJ 222821 (CPV-2c) obtained from the GenBank.

is in accordance with the result reported by Chinchkar, who also indicated that, CPV-2a was the predominant type in India [23]. They reported that out of twenty two CPV isolates from different parts of India, eighteen belonged to CPV-2a, remaining four belonged to CPV$2 b$ and sixteen of the twenty two isolates had substitution 297-Ser $\rightarrow$ Ala. Nandi also reported the circulation of strain CPV-2a, CPV-2b and CPV-2c in the northern Indian geographical area [24]. Similarly, two New CPV-2b strains were detected indicating the presence of New CPV-2b also in certain locations of Southern India. Alignment of the deduced amino acid sequences of partial VP2 gene of the representative canine parvovirus field isolate/sample JN008391, JN008395 (New CPV 2a), JN008393, JF900762 (New CPV-2b) and the reference strains is shown in Figure-1.

In addition to the nucleotide variations at positions $3675,3685,3698,3908$ and 4062, two additional nonsynonymous mutations were observed in the canine parvovirus sequences under study. At nucleotide position 3756-3758 (amino acid residue 324), variations at $3756(\mathrm{~T} \rightarrow \mathrm{A})$ and $3757(\mathrm{~A} \rightarrow \mathrm{T})$ were observed in sequences JN008387, JF900758, JN008397, JN008383, JN008384, JN008396, JN008386, JF900762, JN008385 and JN008392. This type of mutation was also reported in the CPV isolated in China by Zhang [14]. Horiuchi revealed that the residue 324 was prone to strong positive selection in all carnivores [25]. The residue 324 was adjacent to residue 323 , which affected canine transferrin receptor (TfR) binding and, together with residue 93, determined the canine host range [25]. Therefore, the 324 mutation was likely to have an effect on the parvovirus host range. This mutation resulted in the codon change from TAT $\rightarrow$ ATT, indicating change in amino acid residue from $\mathrm{Tyr} \rightarrow$ Ile. Other one was at nucleotide position 4104 (amino acid residue 440), where variation $(A \rightarrow G)$ was observed in case of all the sequences under study except JN008388, JN008389. This mutation resulted in the codon change from $\mathrm{ACA} \rightarrow \mathrm{GCA}$, revealing change in amino acid residue from $\mathrm{Thr} \rightarrow$ Ala. Similar codon change (GCA) at nucleotide position 4104 was also reported in the CPV isolated in Northern America by Kapil [26]. Therefore these two non-synonymous mutations indicated that the CPV strains were under constant selection pressure and were constantly evoluting which might lead to evolution of newer CPV strains/variants in the future.

In addition to non-synonymous mutations, three synonymous mutations were also observed in the sequences under study. One was at nucleotide position 3824 (amino acid residue 346), where variation $(\mathrm{G} \rightarrow$ A) was observed in sequences JN008388 and JN008389. This mutation resulted in the codon change from GAG $\rightarrow$ GAA, both of them coded for Glutamic acid (Glu). Similar codon (GAA) change at nucleotide 
position 3824 was present in prototype FPV and was also reported in the CPV isolated in India by Chinchkar and Mohanraj [13, 23]. Other one was at nucleotide position 4074 (amino acid residue 430), where variation $(\mathrm{G} \rightarrow \mathrm{A})$ was seen in JN008380, JF900760, JN008381, and JN008382, was also reported in the CPV isolated in Japan by Doki [27]. This mutation resulted in the codon change from TTG $\rightarrow$ TTA, both of them coded for Leucine (Leu). Another one mutation was seen at nucleotide position 4121 (amino acid residue 445), where variation $(\mathrm{T} \rightarrow \mathrm{C}$ ) was observed in JN008388, JN008389, JN008390，JN008391, JF900759, JN008393, JN900761，JN008398, JN008380，JF900760，JN008381，JN008394, JN008395 and JN008382. This particular variation changed the codon from ACT $\rightarrow$ ACC, both of which coded for the same amino acid Threonine (Thr). This synonymous mutation was not observed in any of the previously isolated CPVs except in CPVs isolated from India [13, 23]. All these mutations further confirmed sustained constant evolution of canine parvovirus in the geographical region of South India. Therefore, constant surveillance and monitoring involving larger geographical areas are must to identify the nucleotide variations resulting in change in amino acids leading to any possible antigenic variation between vaccine virus and field strains.

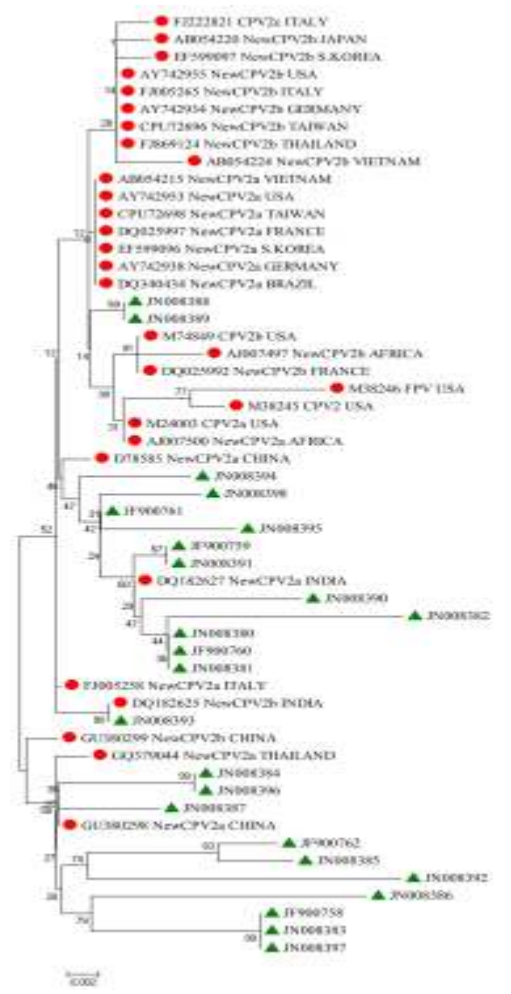

Fig 2. Neighbour J oining with Bootstrap consensus tree (Mega-4.1) constructed using canine parvovirus sequences under study and the reference sequences M38246 (FPV, USA), M38245 (CPV-2,USA), M24003 (CPV-2a, USA), M74849 (CPV-2b, USA), AY742953 (NewCPV-2a, USA), AY742955 (NewCPV-2b, USA), AJ 007500 (CPV2a, Africa), AJ 007497 (CPV-2b, Africa), DQ182627 (NewCPV-2a, India), DQ182625 (NewCPV-2b, India), D78585 (NewCPV-2a, Japan), AB054220 (NewCPV-2b, Japan), AY742938 (NewCPV-2a, Germany), AY742934 (NewCPV-2b, Germany), FJ 005258 (NewCPV2a, I taly), FJ 005265 (NewCPV-2b, I taly), FJ 222821 (CPV-2c, I taly) DQ340434 (NewCPV-2a, Brazil), AB054215 (NewCPV$2 a, V i e t n a m), A B 054224$ (NewCPV-2b, Vietnam), CPU72698
(NewCPV-2a, Taiwan), CPU72696 (NewCPV-2b, Taiwan), EF599096 (NewCPV-2a, South Korea), EF599097 (NewCPV-2b, South Korea), GU380298 (NewCPV-2a, China), GU380299 (NewCPV-2b, China), GQ379044 (NewCPV-2a, Thailand), FJ869124 (NewCPV2b,Thailand), DQ025997 (NewCPV-2a, France), DQ025992 (NewCPV-2b, France)

Phylogenetic analysis of nucleotide sequences of CPV obtained in this study with thirty reference CPV strains from various parts of the world using neighborjoining method with Bootstrap consensus tree (Fig.-2) revealed that one of the field isolates JN008393 was found to be phylogenetically closely related (with bootstrap value of 80) to New CPV-2b strains of India. The sequence JN008394, JN008398, JN008395, JN008391 and JF900761 was closely related to New CPV-2a strain from Japan. The sequence JF900762, JN008385，JN008392，JN008386，JN008383, JN008397, JF900758, JN008384, JN008396 and JN008387 were clustered phylogenetically with New CPV-2a from China and Thailand The remaining sequence JN008390, JN008382 JN008381, JN008380 and JF900760 were phylogenetically related to New CPV-2a strain from India. Phylogenetic analysis revealed that one of the isolates was found to be phylogenetically closely related to New CPV-2b strains of India and most of the sequences under this study were clustered together showing distinct lineage but share ancestral origin with New CPV-2a reference strains of Japan, China, Thailand and India.

\section{Conclusion}

In this study, New CPV-2a (297-Ser $\rightarrow$ Ala) was found to be the predominant strain prevalent in different cities across five Southern Indian states/ Union territories. There was also enough indication of circulation of New CPV-2b strain from different states of Southern India. As the CPV strains were found to be under immense selection pressure and realizing the constant mutations happening in the field strains, continuous monitoring and detection of genetic variations and antigenic changes will be of utmost importance to control CPV infections. As there were reports of occurrence of CPV in vaccinated pups under this study, cross antigenic evaluation of CPV vaccines and field strains is needed for evaluating the real efficacy of CPV vaccines available in the market. The major share of CPV positive cases in this study belonged to unvaccinated dog population, so there is a need for improving public awareness about vaccination of pets against CPV infection.

\section{Authors' contributions}

VMVS and HKM were involved in the design of this research. The research was done by VMVS. HKM has monitored all the activities being a supervisor. HKM and JT have helped in sequence analysis and phylogenetic tree analysis. All authors drafted and revised the manuscript. All authors read and approved the final manuscript.

\section{Acknowledgements}

The authors are grateful to the Dean, Rajiv 
Gandhi College of Veterinary and Animal sciences, Kurumbapet, Pondicherry, India for providing necessary facilities and fund to conduct this research.

\section{Competing interests}

The authors declare that they have no competing interests.

\section{References}

1. Hoelzer, K. and Parrish, C.R. (2010) The emergence of parvoviruses of carnivores, Vet. Res, 41:39.

2. Touihri, L., Bouzid, I., Daoud, R., Desario, C., El Goulli, A.F., Decaro, N., Ghorbel, A., Buonavoglia, C. and Bahloul, C. (2009) Molecular characterization of canine parvovirus-2 variants circulating in dogs in Tunisia, Virus Genes, 38: 249258.

3. Mochizuki, M., Ohshima, T., Une, Y. and Yachi, A. (2008) Recombinant between vaccine and field strains of canine parvovirus is revealed by isolated of virus in canine and feline cell cultures, J Vet Med Sci, 70: 1305-1314.

4. Ozkul, A., Keles, I., Karaoglu, T., Cabalar, M. and Burgu, I. (2002) Detection and RFLP analysis of canine parvovirus (CPV) DNA by polymerase chain reaction (PCR) in a dog, Turk J Vet Sci, 26: 1201-1203.

5. Parrish, C.R. and Carmichael, L.E. (1983) Antigenic structure and variation of canine parvovirus type-2, feline panleukopenia virus, and mink enteritis virus, Virology, 129: 401-414.

6. Strassheim, M.L., Gruenberg, A., Veijalainen, P., Sgro, J.Y. and Parrish, C.R. (1994) Two dominant neutralizing antigenic determinants of canine parvovirus are found on the threefold spike of the virus capsid, Virology, 198: 175-184.

7. Park, S.A., Park, S.Y., Song, C.S., Choi, I.S., Kim, H.Y., Lee, J.B. and Lee, N.H. (2012) Development of a novel vaccine against canine parvovirus infection with a clinical isolate of the type 2b strain, Clin Exp Vaccine Res, 1(1):70-6.

8. Filipov, C., Decaro, N., Desario, C., Amorisco, F., Sciarretta, R. and Buonavoglia, C. (2011) Canine parvovirus epidemiology in Bulgaria, J Vet Diagn Invest, 23:152-154.

9. Kang, B.K., Song, D.S., Lee, C.S., Jung, K., Park, S.J., Kim, E.M. and Park, B.K. (2008) Prevalence and genetic characterization of canine parvoviruses in Korea, Virus Genes, 36: 127-133.

10. Ohshima, T., Hisaka, M., Kawakami, K., Kishi, M., Tohya, Y. and Mochizuki, M. (2008) Chronological analysis of canine parvovirus type 2 isolates in Japan, J Vet Med Sci, 70: 769-775.

11. Ntafis, V., Xylouri, E., Kalli, I., Desario, C., Mari, V., Decaro, N. and Buonavoglia, C. (2010) Characterization of Canine parvovirus 2 variants circulating in Greece, $J$ Vet Diagn Invest, 22:737-740.

12. Buonavoglia, C., Martella, V., Pratelli, A., Tempesta, M., Cavalli, A., Buonavoglia, D., Bozzo, G., Elia, G., Decaro, N. and Carmichael ,L. (2001) Evidence for evolution of canine parvovirus type 2 in Italy, J Gen Virol, 82: 3021-3025.

13. Mohanraj, J., Mukhopadhyay, H.K., Thanislass, J., Antony, P.X. and Pillai, R.M. (2010) Isolation, molecular characterization and phylogenetic analysis of canine parvovirus, Infect Genet Evol, 10: 1237-1241.

14. Zhang, R., Yang, S., Zhang, W., Zhang, T., Xie, Z., Feng, H.,
Wang ,S. and Xia, X. (2010) Phylogenetic analysis of the VP2 gene of canine parvoviruses circulating in China, Virus Genes, 40(3):397-402.

15. Decaro, N., Martella, V., Desario, C., Bellacicco, A.L., Camero, M., Manna, L., d'Aloja, D. and Buonavoglia, C. (2006) First detection of canine parvovirus type 2c in pups with haemorrhagic enteritis in Spain, $J$ Vet Med B Infect Dis Vet Public Health, 53: 468-472.

16. Hirayama, K., Kano, R., Hosokawa-Kanai, T., Tuchiya, K., Tsuyama, S., Nakamura, Y., Sasaki, Y. and Hasegawa, A. (2005) VP2 gene of a canine parvovirus isolate from stool of a puppy, J Vet Med Sci, 67: 139-143.

17. Tamura, K., Dudley, J., Nei, M. and Kumar, S. (2007) MEGA4 :molecular evolutionary genetics analysis (MEGA) software version 4.0, Mol Biol Evol, 24: 1596-1599.

18. Xu, J., Guo, H.C., Wei, Y.Q., Shu, L., Wang, J., Li, J.S., Cao, S.Z. and Sun, S.Q. (2013) Phylogenetic Analysis of Canine Parvovirus Isolates from Sichuan and Gansu Provinces of China in 2011, Transbound Emerg Dis, doi: 10.1111/ tbed. 12078.

19. Parthiban, S., Mukhopadhyay, H.K., Panneer, D., Antony, P.X. and Pillai, R.M. (2011) Isolation and typing of canine parvovirus in CRFK cell line in Puducherry, South India, Indian J Microbiol, 51(4): 456-460.

20. Stepita, M. E., Bain, M. J. and Kass, P. H. (2013) Frequency of CPV Infection in Vaccinated Puppies that attended Puppy Socialization Classes, Journal of the American Animal HospitalAssociation 49:95-100.

21. Nandi, S., Anbazhagan, R. and Kumar, M. (2010) Molecular characterisation and nucleotide sequence analysis of canine parvovirus strains in vaccines in India, Veterinaria Italiana, 46(1):69-81.

22. Martella, V., Cavalli, A., Decaro, N., Elia, G., Desario, C., C,ampolo M., Bozzo, G., Tarsitano, E. and Buonavoglia, C. (2005) Immunogenicity of an intranasally administered modified live canine parvovirus type $2 \mathrm{~b}$ vaccine in pups with maternally derived antibodies, Clin Diagn Lab Immunol, 12: 1243-1245.

23. Chinchkar, S.R., Mohana Subramanian, B., Hanumantha Rao, N., Rangarajan, P.N., Thiagarajan, D. and Srinivasan, V.A. (2006) Analysis of VP2 gene sequences of canine parvovirus isolates in India, Arch Virol, 151: 1881-1887.

24. Nandi, S., Chidri, S., Kumar, M. and Chauhan, R.S. (2010) Occurrence of canine parvovirus type $2 \mathrm{c}$ in the dogs with haemorrhagic enteritis in India, Res Vet Sci, 88: 169-171.

25. Horiuchi, M., Goto, H., Ishiguro, N. and Shinagawa, M. (1994) Mapping of determinants of the host range for canine cells in the genome of canine parvovirus using canine parvovirus/ mink enteritis virus chimeric virus, J Gen Virol, 75: 13191328.

26. Kapil, S., Cooper, E., Lamm, C., Murray, B., Rezabek, G., Johnston, L., Campbell, G. and Johnson, B. (2007) Canine parvovirus types $2 \mathrm{c}$ and $2 \mathrm{~b}$ circulating in North American dogs in 2006 and 2007, J Clin Microbiol, 45: 4044-4047.

27. Doki, M., Fujita, K., Miura, R., Yoneda, M., Ishikawa, Y., Taneno, A. and Kai, C. (2006) Sequence analysis of VP2 gene of canine parvovirus isolated from domestic dogs in Japan in 1999 and 2000, Comp Immunol Microbiol Infect Dis, 29: 199-206. 